\title{
Caracterización de la cizalladura en el aeropuerto de Bilbao y evaluación del sistema LLWAS
}

\author{
María Rosa Pons Reynés ${ }^{1}$ (mponsr@aemet.es) \\ Jorge Pérez Ricci² (jorge_pr_94@hotmail.com) \\ María Antonia Benito Peraita ${ }^{3}$ (mbenitop@aemet.es) \\ Margarita Martín Giménez ${ }^{3}$ (mmarting@aemet.es)

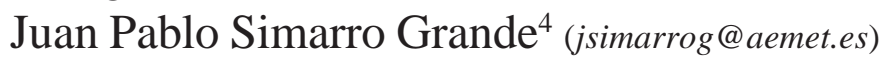 \\ José Lorenzo Lliso Valverde 4 (jllisov@aemet.es) \\ Juan Iglesias Gómez ${ }^{5}$ (jiglesiasg@aemet.es) \\ María López Bartolomé5 (mlopezb@aemet.es) \\ ${ }^{1}$ AEMET / Delegación Territorial en Cantabria \\ ${ }^{2}$ Universidad Politécnica de Madrid / ETS de Ingeniería Aeronáutica y del Espacio \\ ${ }^{3}$ AEMET / Delegación Territorial en el País Vasco \\ ${ }^{4}$ AEMET / Delegación Territorial en la Comunidad Valenciana \\ ${ }^{5}$ AEMET / Dirección de Producción e Infraestructuras / Departamento de Infraestructura y Sistemas
}

\begin{abstract}
RESUMEN
El objetivo del presente estudio es caracterizar la cizalladura del aeropuerto de Bilbao y determinar la capacidad del sistema LLWAS actualmente instalado para detectarla. Mediante la realización de una campaña de datos procedentes de aeronaves se ha podido comprobar que la turbulencia tiene un protagonismo muy importante frente a la cizalladura y determinar los niveles en los que se dan con mayor frecuencia. Así mismo, se ha identificado el patrón de viento dominante (obteniéndose un nuevo resultado de vientos flojos en superficie hasta en un $40 \%$ de los casos) y se ha realizado un análisis preliminar de la capacidad de predicción de la cizalladura del modelo HARMONIE-AROME de 2,5 km de resolución, desarrollando un nuevo producto operativo de secciones verticales.

La campaña de datos ha permitido también concluir que la capacidad de detección del sistema LLWAS en el aeropuerto de Bilbao es baja debido principalmente al predominio de la cizalladura vertical.
\end{abstract}

PALABRAS CLAVE: cizalladura; turbulencia; aeronáutica; LLWAS; observaciones de aeronaves; colaboración con usuarios.

\section{INTRODUCCIÓN}

La cizalladura del viento se define como un cambio en la velocidad y/o dirección del viento en el espacio y puede tener su origen en diversos fenómenos, como por ejemplo las brisas, las tormentas, los sistemas frontales, las corrientes en chorro a baja altura o la orografía. La cizalladura puede producir cambios bruscos en la sustentación de una aeronave por lo que, si bien puede darse en todos los niveles de la atmósfera, su 
ocurrencia en la capa inferior (por debajo de $1600 \mathrm{ft}$ ) es donde tiene mayor adversidad para la aviación (OACI, 2005); en las fases de ascenso y aproximación el piloto dispone de márgenes de maniobra menores para poder corregir la trayectoria de la aeronave.

En el aeropuerto de Bilbao — casi 5 millones de pasajeros en 2017 (AENA, 2017)—, la cizalladura del viento es el fenómeno meteorológico que causa mayor impacto en las aproximaciones de las aeronaves. Según datos de ENAIRE, aproximadamente el $30 \%$ de las maniobras de «motor y al aire» (maniobra en la que el piloto frustra el aterrizaje cuando está en la fase de aproximación final, aumentando la potencia del motor y yéndose nuevamente al aire) ocurridas en 2017 fueron debidas a la cizalladura. Si se consideran también las clasificadas como debidas a la turbulencia (cambios bruscos en las componentes horizontal y vertical del viento que provocan remolinos y un movimiento caótico), se alcanza el 40 \%. La localización geográfica de dicho aeropuerto, en un valle de orientación noroeste-sureste con cumbres cercanas a $1000 \mathrm{~m}$ a menos de $12 \mathrm{~km}$ de distancia hacia el suroeste, es la causa de que se produzcan dichos fenómenos cuando el flujo sinóptico es de componente sur y el aeropuerto se encuentra a sotavento de la cadena montañosa (fíg. 1).

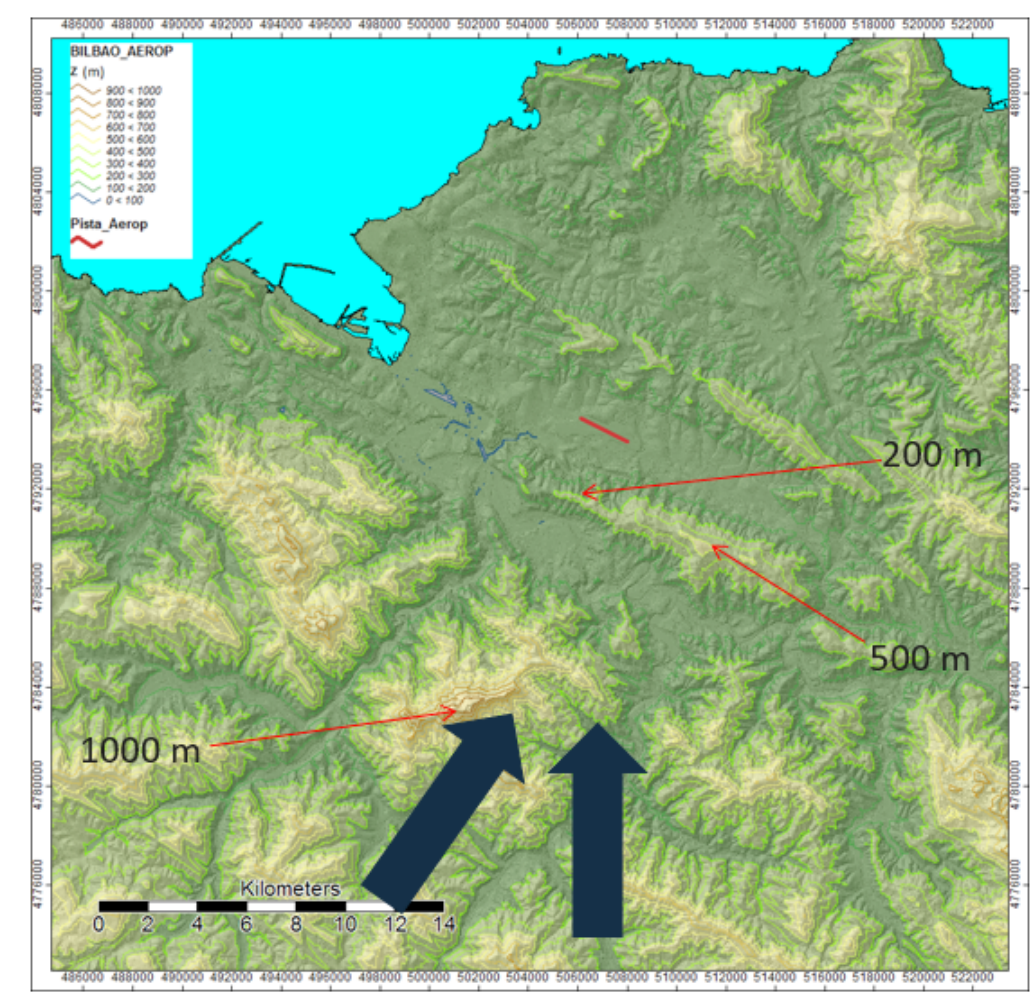

Figura 1. Relieve en el entorno del aeropuerto de Bilbao (en rojo, la pista principal). Las flechas gruesas indican la dirección del viento sinóptico en la mayoría de los casos de cizalladura y turbulencia.

Con el objetivo de contribuir a la mejora de la seguridad de las operaciones aéreas, la Agencia Estatal de Meteorología (AEMET) instaló en dicho aeropuerto un sistema de detección de cizalladura de niveles bajos denominado LLWAS (Low Level Windshear Alert System), que empezó a funcionar en 2008; dicho sistema está instalado únicamente en dos aeropuertos españoles: Bilbao y Tenerife Sur.

La falta de confianza en el sistema de Bilbao ante el aparente elevado número de falsas alarmas motivó que en abril de 2016, y en el marco del convenio entre el Colegio Oficial de Pilotos (COPAC) y AEMET, ambas instituciones incluyeran como línea de actuación «mejorar el uso de la información suministrada por el sistema LLWAS de Bilbao para la identificación de cizalladura, desarrollando los procedimientos necesarios». Siguiendo el ejemplo del aeropuerto de Tenerife Sur (VERnière, 2014), en el que las alarmas del LLWAS están incorporadas a nivel operativo en los procedimientos de la torre de control y de AEMET, fruto de un estudio y una 
estrecha colaboración entre todos los agentes implicados, en septiembre de 2016 se celebró una reunión con los usuarios en el aeropuerto de Bilbao. Asistieron representantes de AEMET, AENA (gestor de aeropuertos), ENAIRE (gestor de navegación aérea), COPAC, APROCTA (Asociación Profesional de Controladores de Tránsito Aéreo) y compañías aéreas, y en ella se definieron los objetivos del proyecto: caracterizar mejor los fenómenos de cizalladura y turbulencia, y determinar la capacidad del LLWAS para detectarla.

\section{SISTEMA LLWAS}

El sistema LLWAS está formado por diez torres situadas alrededor de la pista con sensores ultrasónicos de velocidad y dirección del viento, a alturas que varían entre los $11 \mathrm{y}$ los 22 metros en el caso de Bilbao (véase figura 2). Dicho sistema fue desarrollado por la Administración Federal de Aviación de Estados Unidos (FAA) que estableció en un extenso documento (FAA, 1989) todos los criterios que debían seguirse en el diseño de la instalación (distancias entre sensores y a la pista, ángulos, altura de los sensores, etc). El algoritmo del sistema (desarrollado en origen por el Centro Nacional de Investigación Atmosférica, NCAR) calcula las divergencias y

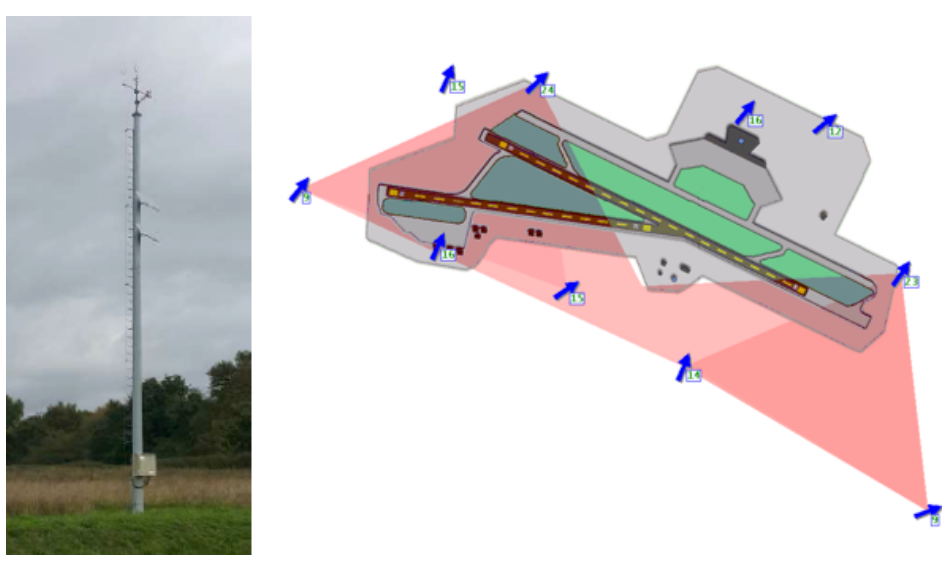

Figura 2. Detalle de una de las diez torres del sistema LLWAS de Bilbao y ejemplo de visualización de sus datos en la oficina meteorológica del aeropuerto (OMA). convergencias entre sensores que traduce en ganancias o pérdidas de la velocidad del viento de cara que sufriría la aeronave, emitiendo alarmas a partir de $15 \mathrm{kt}$. El sistema tiene un alcance máximo de 1 milla náutica, y sus datos se actualizan cada 10 segundos.

El sistema emite alarmas que clasifica como microrreventón MB (para pérdidas superiores a $30 \mathrm{kt}$ de viento en cara) o como cizalladura WSA (para ganancias superiores a 15 kt o pérdidas entre 15 y $30 \mathrm{kt}$ ). Además de la pérdida o ganancia de viento en cara, el sistema especifica la cabecera en la que se produce la alarma, la zona (sobre la pista o a una milla de distancia) y la dirección y la velocidad del viento en dicha cabecera. Un ejemplo de mensaje de alarma sería el siguiente: 12A WSA 15k+ RWY 160 08. Si el personal de la OMA considera que la alarma es falsa, puede desactivar el envío de la misma a la torre de control.

En otoño de 2016 AEMET realizó una revisión en profundidad del sistema. Por un lado, se revisaron las infraestructuras, renovando sensores y baterías, analizando los posibles apantallamientos por el entorno, realizando estudios de cobertura ante fallos eventuales de sensores y revisando los criterios que se cumplían del documento de la FAA; de dicha revisión surgieron una serie de recomendaciones. Por otro lado, se plantearon una serie de mejoras en el software, que consistieron principalmente en la implementación de filtros en la velocidad del viento (tanto de valor máximo como de coherencia entre sensores) reduciendo considerablemente las falsas alarmas emitidas por el sistema (PÉREZ, 2017).

\section{CAMPAÑA EXPERIMENTAL}

Además de la revisión del LLWAS, en la reunión con los usuarios se acordó la realización de una campaña de recogida de medidas, que tuvo lugar entre octubre de 2016 y mayo de 2017, coincidiendo con la época del año en la que los fenómenos de cizalladura y turbulencia se dan con mayor frecuencia. Se solicitó la colaboración de las compañías aéreas para que, por un lado, sus pilotos proporcionaran a la torre de control datos de cizalladura, turbulencia y viento en sus aproximaciones y despegues de Bilbao y, por otro lado, suministraran los datos meteorológicos automáticos registrados por sus aviones en una serie de días concretos. 
Sobre esta última cuestión se prestó a colaborar la compañía Vueling, que cedió los datos registrados en 139 vuelos de dicho periodo (datos de los últimos $5000 \mathrm{ft}$ cada 4 segundos), y Lufthansa, que proporcionó una base de datos de viento de aproximadamente 6000 vuelos de años anteriores (2009-2016) en 17 niveles de 30 a $5000 \mathrm{ft}$. Estos últimos datos no tenían identificada la fecha pero permitieron extraer conclusiones generales sobre el flujo de viento en Bilbao.

Así mismo, en el proyecto se analizaron también los datos de viento obtenidos a través del programa E-AMDAR de la Organización Meteorológica Mundial (WMO, 2017), que consiste en la recogida de datos meteorológicos medidos de forma automática por las aeronaves y su posterior envío a los Servicios Meteorológicos Nacionales para que puedan ser asimilados en sus modelos numéricos. De las compañías aéreas que operan en el aeropuerto de Bilbao, participan en dicho programa Air France, British Airways, Easy Jet, Eurowings, Germanwings, KLM y Lufthansa, disponiéndose de datos de 129 vuelos en el periodo de interés. La resolución temporal de dichos datos es menor, obteniéndose aproximadamente información de 4 niveles por debajo de $2000 \mathrm{ft}$.

Adicionalmente, en el presente estudio se analizaron datos de superficie, datos proporcionados por AENA y ENAIRE y salidas de modelos numéricos. En concreto, se desarrolló un producto específico de secciones verticales paralelas y perpendiculares a la pista a partir del modelo de mesoescala HARMONIE-AROME de 2,5 km de resolución, representándose el viento, la temperatura potencial y la energía cinética turbulenta. Todo ello supuso un gran volumen de datos de naturaleza muy diversa. Se detallan a continuación todos los datos utilizados en el proyecto:

- Datos de superficie: alarmas del sistema LLWAS, incidencias de dicho sistema anotadas por el personal de la OMA, informes METAR y datos de viento diezminutales de las cabeceras 12 y 30.

- Datos de altura: datos de viento obtenidos del programa E-AMDAR, datos meteorológicos registrados por las aeronaves de Vueling y datos de pilotos recogidos por la torre de control. Además, datos registrados por las aeronaves de Lufthansa de un periodo diferente (2009-2016) pero sin la identificación de la fecha.

- Otros datos: mensajes ATIS, registro de aterrizajes frustrados (proporcionados por AENA y ENAIRE), predicciones del modelo HARMONIE-AROME de 2,5 km de resolución y análisis del modelo HRES-IFS del ECMWF de $16 \mathrm{~km}$.

\section{RESULTADOS}

\subsection{Cizalladura y turbulencia}

En relación con los datos proporcionados por los pilotos sobre cizalladura, turbulencia y viento, y anotados por la torre de control, provenientes de 133 vuelos de 22 compañías aéreas, la distribución en las cabeceras es desigual, obteniéndose aproximadamente un $80 \%$ de datos con la cabecera 12 operativa frente al $20 \%$ de la cabecera 30 . El $60 \%$ de los datos se notificó en aproximaciones y el $40 \%$ en despegues.

Del conjunto de notificaciones recibidas, destaca el hecho de que el $80 \%$ de los vuelos notificó turbulencia (con el mayor número de reportes entre 2500-3000 ft, fig. 3) frente al $30 \%$

Figura 3.

Distribución de las notificaciones de cizalladura (WS) y turbulencia (TURB) de los pilotos en función de la altura.

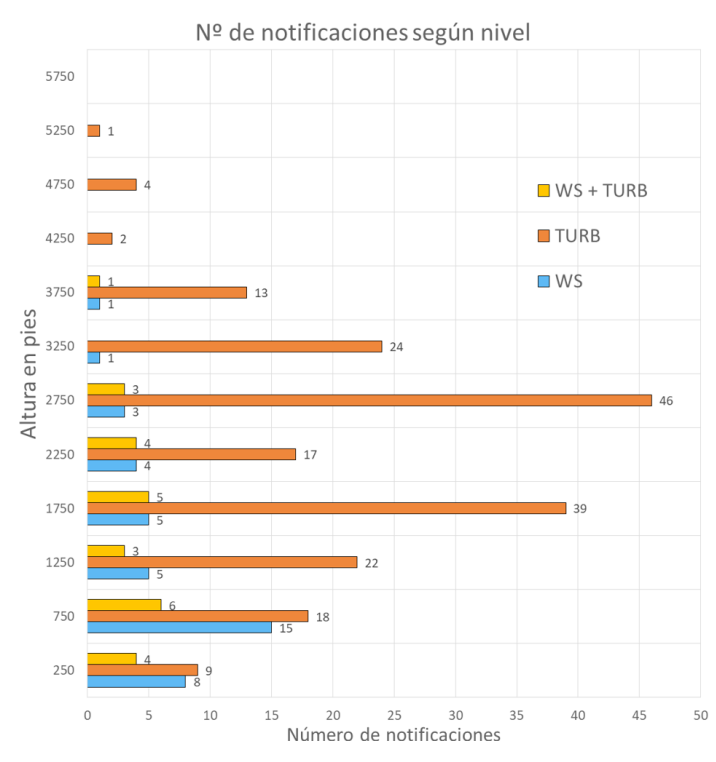




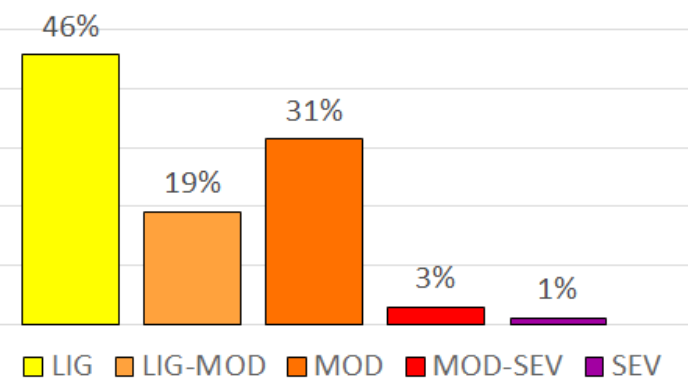

Figura 4. Notificaciones de turbulencia de los pilotos en función de su intensidad.

que notificó cizalladura (mayor número de reportes entre 500-1000ft). El $45 \%$ de estos últimos indicó el signo de la cizalladura, predominando en un $66 \%$ de los casos la cizalladura positiva.

De los 105 vuelos con turbulencia notificada, aproximadamente el $35 \%$ la reportaron con intensidad moderada o severa, si bien cabe recordar que la apreciación de la intensidad es una cuestión subjetiva del piloto. En conjunto predominaron los reportes de intensidad ligera con un $45 \%$ (fig. 4), salvo en las aproximaciones por la cabecera 30 en las que predominó la intensidad moderada ( $38 \%$ frente a $31 \%$ de intensidad ligera, no mostrado aquí). Los reportes de mayores intensidades (moderada-severa o severa) se produjeron entre 1500 y $3500 \mathrm{ft}$.

\subsection{Patrones de viento}

A partir de un análisis conjunto de los datos, se ha podido comprobar que la configuración sinóptica más habitual en niveles bajos en los casos de cizalladura y turbulencia es un centro profundo de bajas presiones al norte de la zona (ver fig. 5.d) que produce un flujo sinóptico del suroeste fuerte.

Al estar el aeropuerto situado a sotavento de la cadena montañosa, el flujo en superficie se ve afectado por la orografía, debiendo remontar la cadena o bordearla. El caso más común es el desvío del viento, soplando del sur-sureste sobre el aeropuerto (fig. 5.a), seguido en segundo lugar por un viento en superficie que se mantiene del suroeste (fig. 5.b); no se aprecian diferencias significativas en cuanto a su intensidad. En algunos casos muy puntuales (comprobados únicamente con los datos de Lufthansa), se ha podido observar que el flujo es desviado en superficie a viento del noroeste (fig. 5.c).
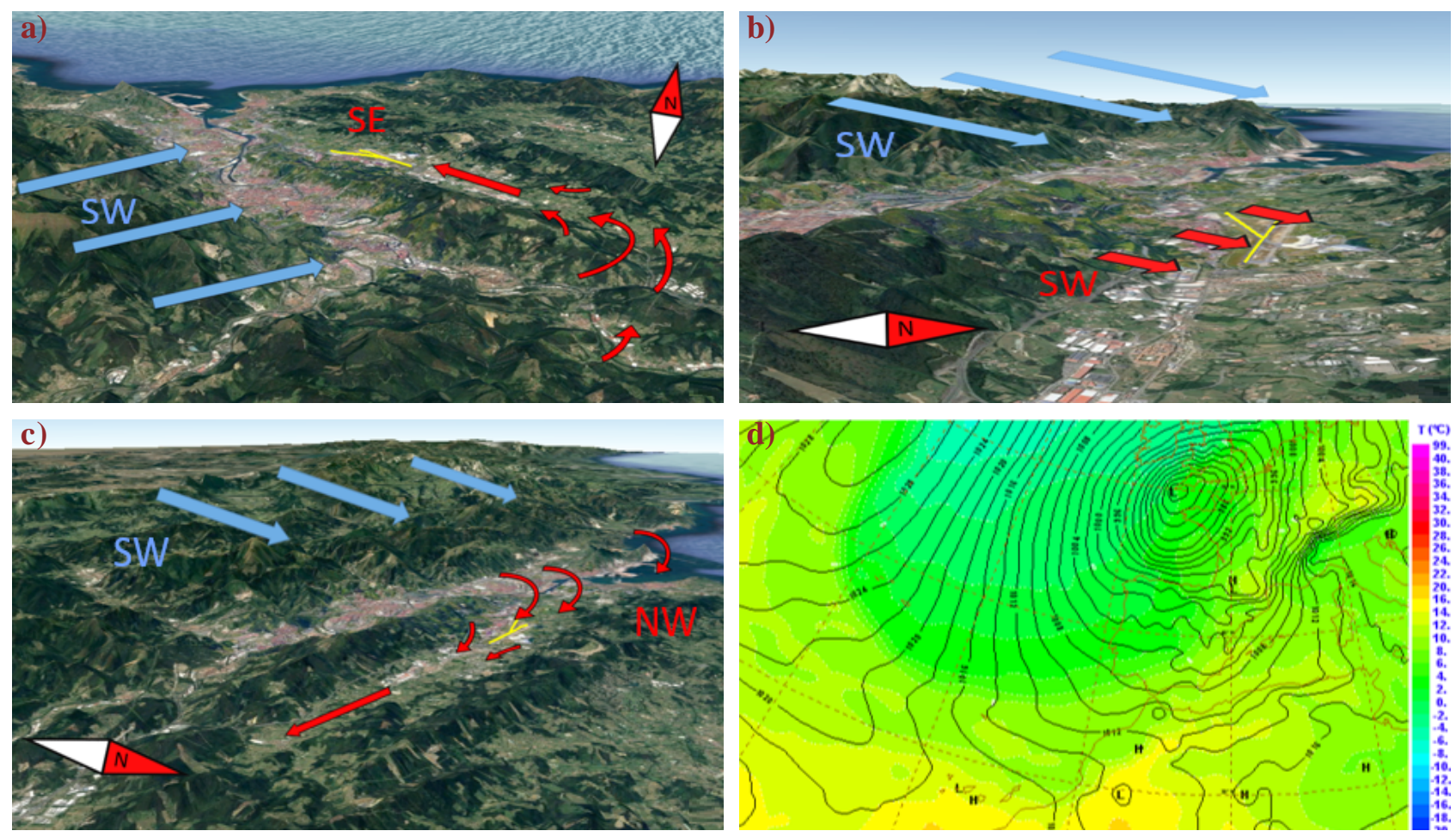

Figura 5. En las tres primeras figuras, viento observado sobre el aeropuerto (flechas rojas), con flujo sinóptico fuerte del suroeste en niveles altos (flechas azules); en amarillo las pistas del aeropuerto.

a) Viento del sureste —el más habitual—; b) suroeste y c) noroeste — muy poco común—;

d) ejemplo de configuración sinóptica más común en niveles bajos, representando la presión a nivel del mar (isolíneas) y la temperatura en $850 \mathrm{hPa}$ (en colores). 
Si analizamos la relación de los casos de cizalladura, turbulencia y aterrizajes frustrados con el viento en superficie (fig. 6), se observa que en los casos de cizalladura predomina el viento del sureste, en los de turbulencia, el viento del sur y sureste, y en los aterrizajes frustrados predomina claramente el suroeste (viento cruzado a la pista).
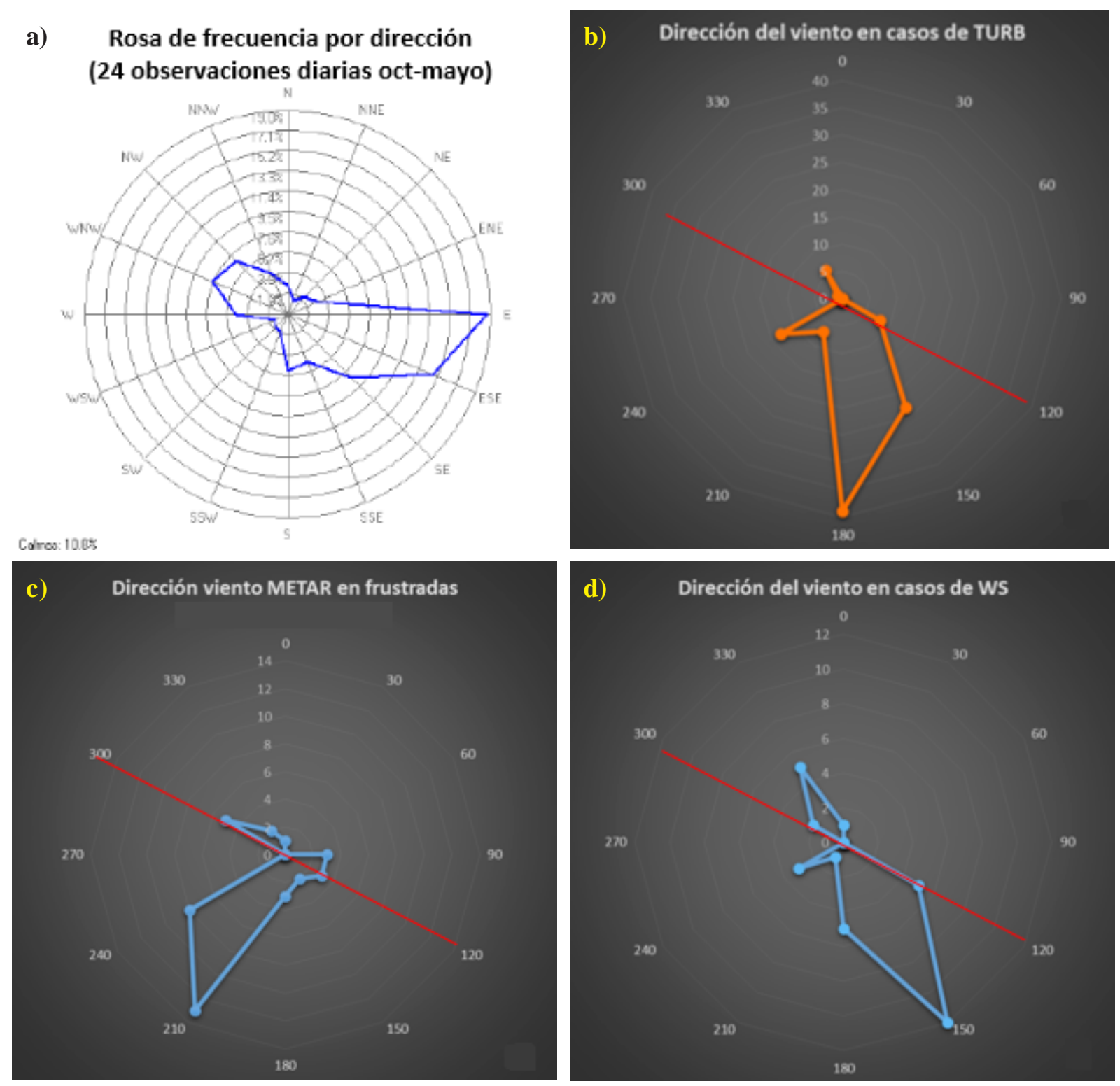

Figura 6. Rosa de frecuencia de la dirección del viento en: a) el periodo completo —octubre de 2016 a mayo de 2017 — , considerando observaciones horarias; b) los casos de notificaciones de turbulencia;

c) los aterrizajes frustrados; y d) las notificaciones de cizalladura.

Las tres últimas figuras se han realizado a partir del viento en el METAR.

Otro de los resultados importantes del estudio es que se ha podido comprobar que en aproximadamente el $40 \%$ de los casos de cizalladura y turbulencia, y de aterrizajes frustrados por dichas causas, el viento era flojo en la pista. Es por ello que es de gran importancia la información que proporcionan los pilotos en sus aproximaciones para que la torre pueda informar a los siguientes vuelos y estén prevenidos; además, es importante que la torre comunique los reportes de cizalladura a la OMA para que se incluya el fenómeno en el informe METAR y así los pilotos dispongan de dicha información antes del inicio del vuelo y puedan tomar las medidas preventivas oportunas. Los informes TAF no incluyen una predicción de cizalladura y, por tanto, su detección local en Bilbao se reduce a los informes de pilotos o al sistema LLWAS. 


\subsection{Estudio con las salidas del modelo HARMONIE-AROME}

Los datos automáticos proporcionados por la compañía Vueling y los datos del programa E-AMDAR han permitido validar de forma preliminar y cualitativa la salida del modelo numérico HARMONIE-AROME de 2,5 km de resolución; por ejemplo, el modelo predice bien el giro del viento a sur-sureste en superficie que se produce en la mayoría de los casos (fig. 7).

a)

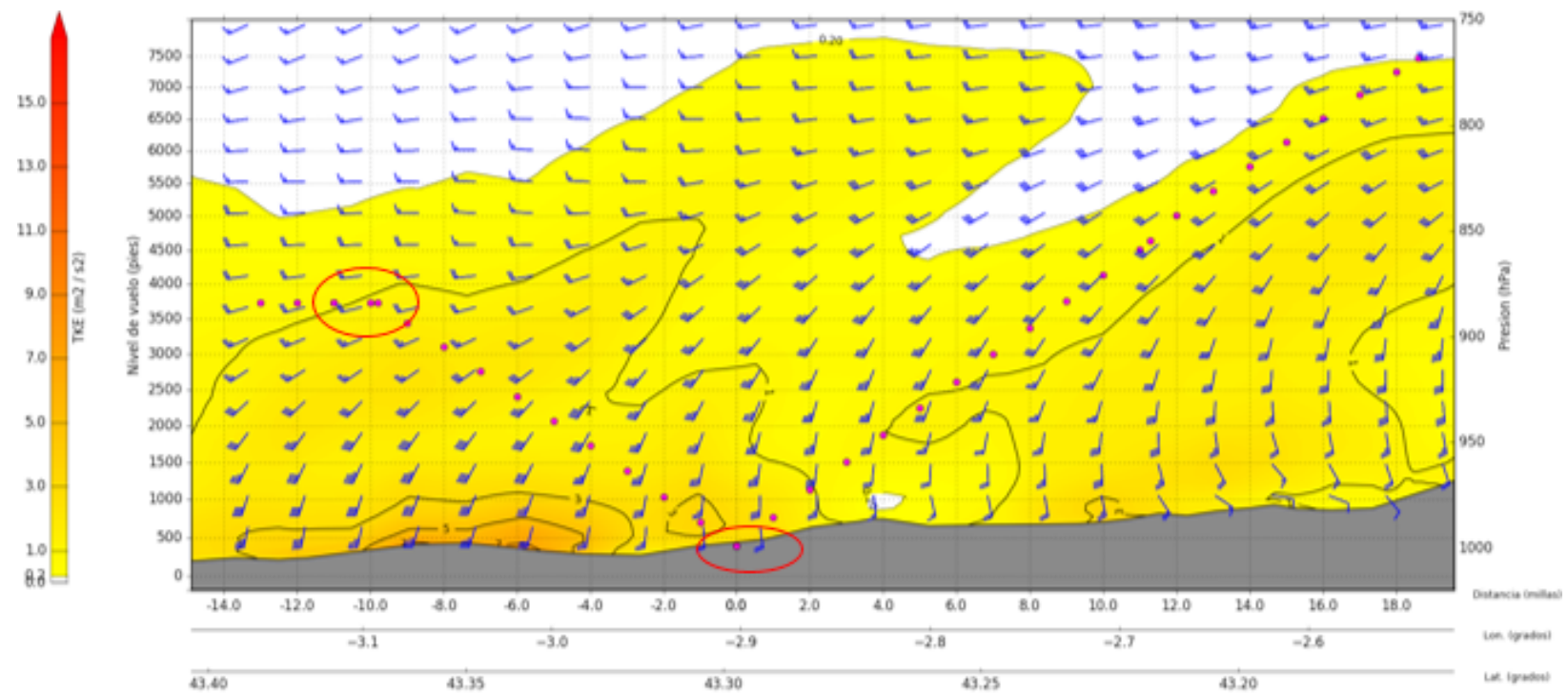

b)

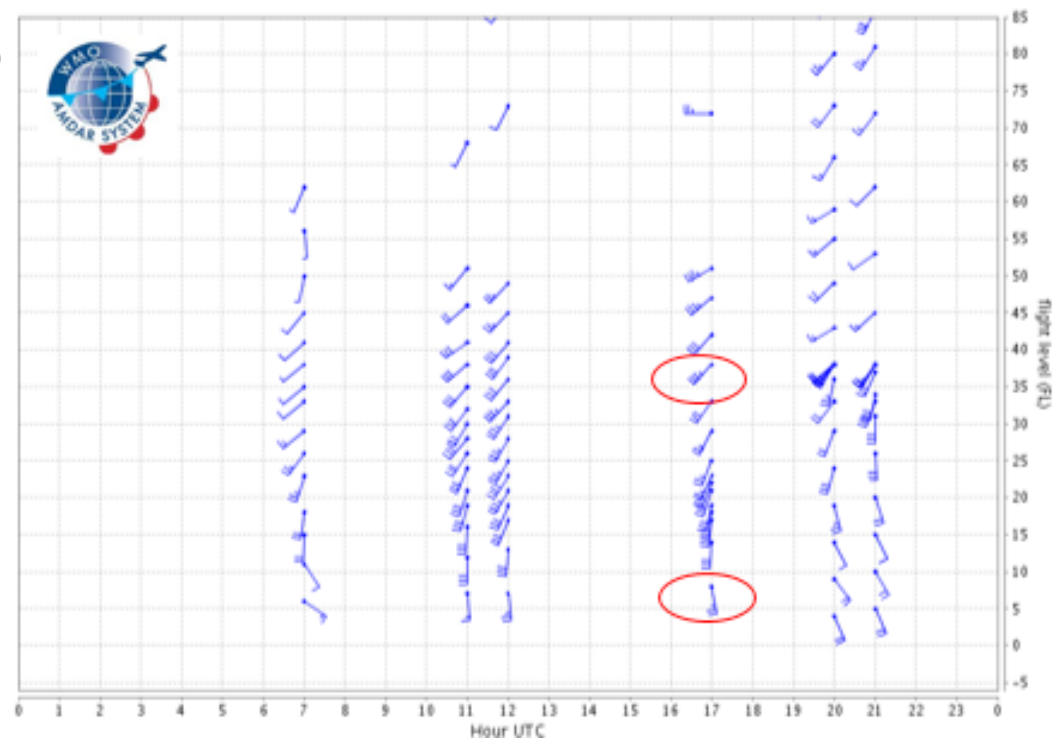

Figura 7. a) Corte vertical del viento (flechas) y la energía cinética turbulenta (en colores) sobre el eje de la pista, a partir del modelo HARMONIE-AROME; en puntos rosas la senda de planeo.

b) Perfiles verticales del viento en 6 vuelos diferentes (hora UTC en el eje horizontal) en ascenso o descenso de Bilbao obtenidos de los datos E-AMDAR.

Se ha podido comprobar también que no hay un patrón homogéneo de cizalladura en las aproximaciones a Bilbao, a diferencia de lo que ocurre en Tenerife Sur. Si bien en líneas generales, la velocidad del viento disminuye a medida que el avión desciende, esta disminución no se produce de forma suave sino que las aeronaves sufren grandes variaciones de velocidad (tanto aumento como disminución) en las aproximaciones, y en algunas ocasiones también de dirección. En algunos casos el modelo muestra unos máximos de velocidad en altura sobre el aeropuerto, cuya posición varía con el tiempo y que son corroborados por los datos de Vueling (fig. 8). 

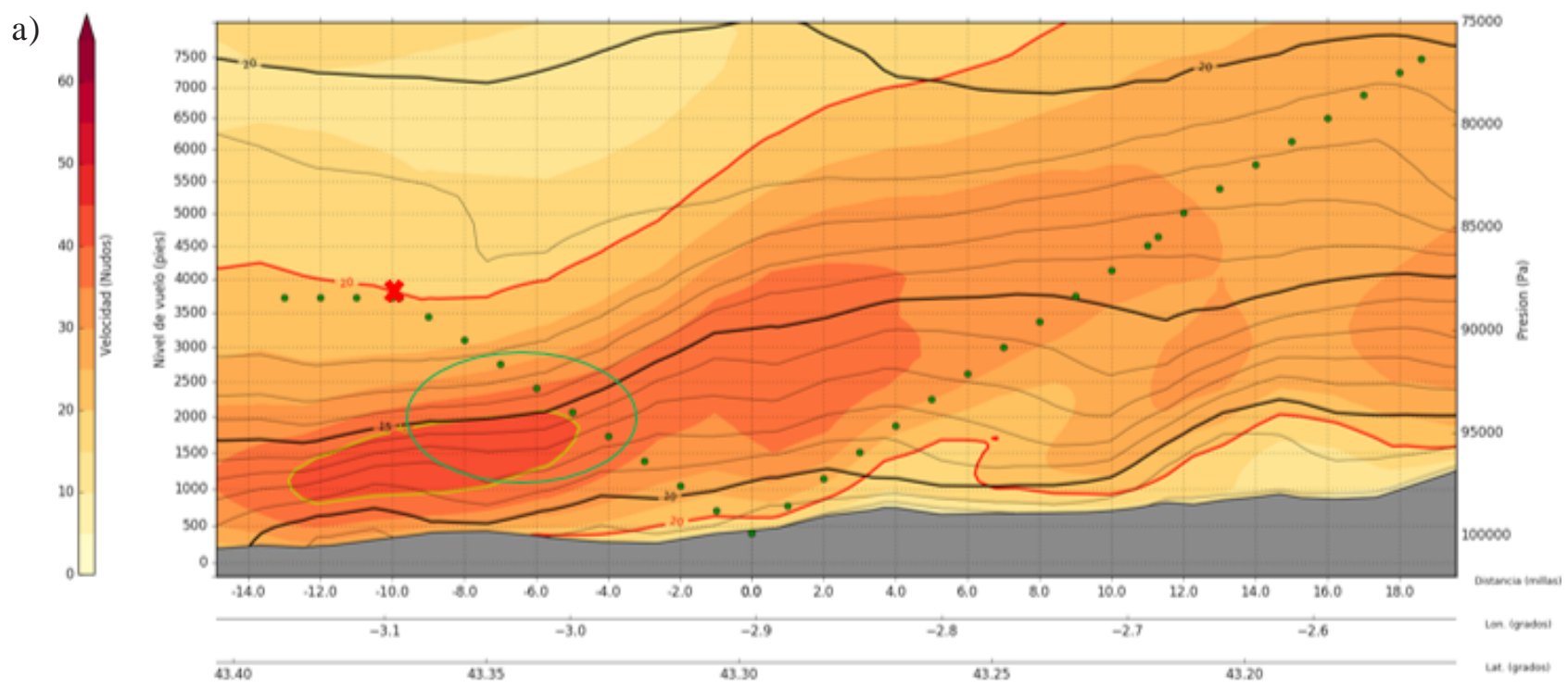

b)

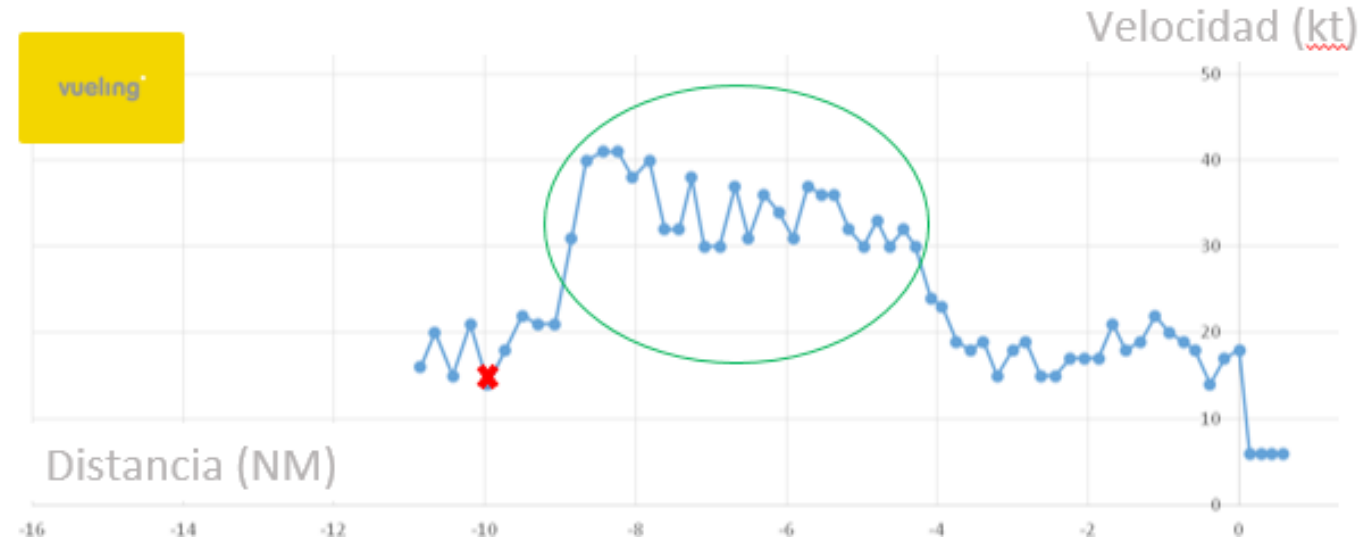

Figura 8. a) Corte vertical del viento (en colores) y la temperatura potencial (isolíneas)

sobre el eje de la pista, a partir del modelo HARMONIE-AROME; en puntos verdes la senda de planeo.

b) Velocidad del viento en nudos registrada por un avión de la compañía Vueling en su aproximación a Bilbao

(distancia decreciente a la zona de toma de contacto en el eje horizontal), a la misma hora.

\subsection{Alarmas del sistema LLWAS}

En la verificación llevada a cabo de las alarmas del sistema se han analizado aproximadamente 47000 mensajes (el sistema mide con una frecuencia de diez segundos), encontrándose que en el $65 \%$ de los casos la duración de las alarmas es inferior a cinco minutos. Si bien se ha logrado disminuir considerablemente las falsas alarmas tras la implementación de una serie de filtros en la velocidad del viento, se considera que siguen siendo importantes; en ello influye probablemente la orografía compleja del entorno que produce un flujo de viento irregular y turbulento sobre el aeropuerto.

A partir de los datos de pilotos y de aterrizajes frustrados obtenidos en la presente campaña, se ha podido comprobar que su capacidad de detección en el aeropuerto de Bilbao es solo de aproximadamente el $50 \%$, debido, entre otras cosas, a que el sistema no está diseñado para detectar la cizalladura vertical (la más común en Bilbao según los datos analizados).

\section{CONCLUSIONES}

La campaña de datos realizada en el presente proyecto ha permitido caracterizar mejor los fenómenos de cizalladura y turbulencia en el aeropuerto de Bilbao. Los datos proporcionados por los pilotos (con la 
colaboración de la torre de control) y las compañías aéreas han permitido comprobar que la turbulencia tiene un protagonismo muy importante en Bilbao ya que está presente en el $80 \%$ de las notificaciones recibidas (con un máximo de reportes en el nivel de 2500-3000 ft), frente al $30 \%$ de cizalladura (máximo de reportes en 500-1000 ft).

El patrón de viento dominante en los días de cizalladura y turbulencia es un flujo sinóptico fuerte del suroeste que se ve desviado por la cadena montañosa cercana al aeropuerto, soplando del sur o sureste sobre la pista; en los casos de aterrizajes frustrados, predomina el viento del suroeste sobre el aeropuerto.

Se ha podido determinar que en aproximadamente el $40 \%$ de los casos de cizalladura, turbulencia o aterrizajes frustrados, el viento en superficie es flojo. Un hallazgo importante que servirá para mejorar la descripción de los fenómenos de viento recogidos en la versión actual de la Publicación de Información Aeronáutica AIP (ENAIRE, 2018) de Bilbao que menciona únicamente vientos moderados o fuertes en los casos de cizalladura y turbulencia.

Así mismo, los datos procedentes de aeronaves han permitido realizar una validación preliminar de los datos de viento del modelo numérico HARMONIE-AROME de 2,5 km de resolución, simulando razonablemente bien la cizalladura vertical que se produce sobre el aeropuerto y que debe seguirse estudiando en el futuro. Además, las secciones verticales aquí desarrolladas se han incorporado como producto operativo en los demás aeropuertos.

Por último, la campaña de datos ha permitido realizar una verificación de las alarmas del sistema LLWAS de Bilbao, obteniéndose una capacidad de detección baja debido principalmente al predominio de la cizalladura vertical en dicho aeropuerto. Se añade el hecho de que el sistema aún presenta un número considerable de falsas alarmas, no está diseñado para detectar la turbulencia y su mantenimiento requeriría una inversión fuerte en infraestructuras debido a su antigüedad, por lo que AEMET ha tomado la decisión de retirar el LLWAS del aeropuerto de Bilbao y apostar por un nuevo sistema: un lidar (Laser Imaging Detection And Ranging) Doppler 3D.

Dicho sistema emite un haz de luz láser infrarrojo y basa su funcionamiento en medir la velocidad del viento a partir del desplazamiento Doppler que sufre la luz dispersada de vuelta hacia el sensor por los aerosoles que están en movimiento en la atmósfera. La característica de poder realizar un escaneo en 3D implica que puede detectar la cizalladura horizontal y vertical, pudiendo realizar escaneos en la propia senda de planeo. El único requisito es que haya suficientes aerosoles y que el aire esté seco, ya que la señal presenta importantes problemas de apantallamiento con nubosidad o precipitación; en el estudio realizado se ha podido comprobar que en Bilbao la mayoría de los fenómenos de cizalladura y turbulencia ocurren en aire seco.

El lidar Doppler 3D ha sido instalado en diversos aeropuertos como Hong Kong (Shun y CHAN, 2008, y Hon y Chan, 2014) o Niza (Augros et al., 2012) habiéndose podido comprobar su eficacia para detectar la cizalladura y la turbulencia. En el caso de que se logre sacar adelante un expediente para su suministro en el aeropuerto de Bilbao, será necesario el desarrollo de un algoritmo para obtener alarmas de cizalladura y turbulencia a partir de la salida de viento, un proceso que es complejo. En ese momento será de nuevo fundamental la colaboración de todos los usuarios para poder realizar otra campaña de medidas que permita validar los datos y obtener así alarmas que puedan ser de utilidad para los pilotos en sus aproximaciones y despegues de Bilbao.

\section{AGRADECIMIENTOS}

Los autores desean agradecer su colaboración al personal de la OMA de Bilbao, por el seguimiento exhaustivo de las alarmas e incidencias del sistema LLWAS durante la campaña; a ENAIRE, AENA, COPAC y APROCTA, por su participación en diversas fases del proyecto y su difusión; a las compañías aéreas Vueling 
y Lufthansa, por suministrar los datos automáticos de sus aviones; a los pilotos de más de veinte compañías aéreas, por proporcionar los datos relevantes en sus maniobras de aproximación y despegue al aeropuerto de Bilbao; a Météo-France y al CNRM (Centre National de Recherches Météorologiques), por su asesoramiento sobre la tecnología lidar; y al Centro Meteorológico de Tenerife y a la OMA de Tenerife Sur, por su transmisión de conocimientos sobre el sistema LLWAS y su apoyo. Por último, un recuerdo especial para nuestro compañero Rafael Vernière Ferrer, responsable de la puesta en marcha operativa del sistema LLWAS en el aeropuerto de Tenerife Sur.

\section{REFERENCIAS}

AENA, 2017. Informe estadístico anual de los aeropuertos españoles.

Augros, C., Tabary, P., Davrinche, D. y Schwartz, E., 2012. Test of an X-band Doppler polarimetric radar combined with a Doppler LIDAR for wind shear detection at Nice Airport. ERAD 2012 - Seventh European Conference on Radar in Meteorology and Hydrology.

ENAIRE, 2018. AIP AD 2 - LEBB, Datos del aeródromo, Apartado 23 de Información Suplementaria.

FAA, 1989. Siting Guidelines for Low Level Windshear Alert Systems (LLWAS) Remote Facilities, 6560.21A.

Hon, K. K. y CHAN, P. W., 2014. Application of LIDAR-derived eddy dissipation rate profiles in low-level windshear and turbulence alerts at Hong Kong International Airport. Meteorol. Appl., 21, 74-85.

OACI, 2005. Manual sobre cizalladura del viento a poca altura, Doc. 9817 - AN/449.

Pérez, J., 2017. Caracterización de la cizalladura y turbulencia del aeropuerto de Bilbao. Trabajo Fin de Grado. Escuela Técnica Superior de Ingeniería Aeronáutica y del Espacio, Universidad Politécnica de Madrid.

Shun, C. M. y Chan, P. W., 2008. Applications of an Infrared Doppler Lidar in Detection of Wind Shear. J. Atmos. Oceanic Technol., 25, 637-655.

VerniÈre, R., 2014. Cizalladura del Aeropuerto de Tenerife Sur. Aviador, 73, 17-18.

WMO, 2017. Guide to Aircraft-based Observations, WMO - No. 1200. 